Title:

\title{
Symptoms of posttraumatic stress disorder and depression among bereaved and non-bereaved survivors following the 2008 Sichuan earthquake
}

Running title: PTSD and depression among bereaved earthquake survivors

Authors:

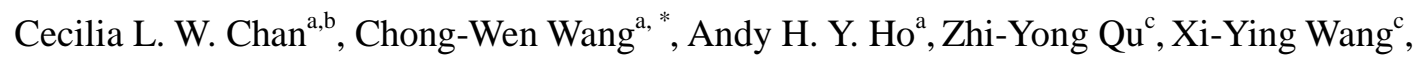
Mao-Sheng Ran ${ }^{\mathrm{d}, \mathrm{e}}$, Wen-Jun Mao ${ }^{\mathrm{f}}$, Ben Qi-Bin Luc ${ }^{\mathrm{c}}$, Braven Qiang Zhang ${ }^{\mathrm{c}}$, Xiu-Lan Zhang ${ }^{\mathrm{c}}$

${ }^{\mathrm{a} C e n t e r ~ o n ~ B e h a v i o r a l ~ H e a l t h, ~ T h e ~ U n i v e r s i t y ~ o f ~ H o n g ~ K o n g, ~} 5$ Sassoon Road, Pokfulam, Hong Kong, China;

${ }^{\mathrm{b}}$ Department of Social Work and Social Administration, The University of Hong Kong, Pokfulam, Hong Kong, China;

'School of Social Development \& Public Policy, Beijing Normal University, Beijing, 100875, China;

${ }^{\mathrm{d}}$ Division of Health Sciences, University of Guam, Guam 96923, USA;

${ }^{\mathrm{e}}$ Department of Psychiatry, West China Hospital, Sichuan University, Chengdu, Sichuan 610041, China;

${ }^{\mathrm{f}}$ Chengdu Mental Health Center, Chengdu, Sichuan 610036, China.

*Corresponding author. Tel: +852 2831 5218; fax: +852 2816 6710;

E-mail address: wangcw@hku.hk 


\begin{abstract}
Many studies have suggested that unexpected death of a loved one is an important risk factor of posttraumatic stress disorder (PTSD) and depression among disaster survivors, but few have examined the magnitude of psychiatric morbidities among bereaved survivors. This study examined the prevalence rates of clinically significant PTSD and depressive symptoms and their associated risk factors among Chinese adult survivors following the 2008 Sichuan earthquake. Two hundred and fifty-one bereaved adults were compared with 1,474 non-bereaved adult survivors. The estimated rates of PTSD and depressive symptoms were $65.6 \%$ and $64.8 \%$ for those who lost first-degree family members, $34.1 \%$ and $45.5 \%$ for those who lost second-degree relatives, and $27.1 \%$ and $37.5 \%$ for non-bereaved survivors respectively. Loss of a child was a significant predictor of psychopathological symptoms. The results suggested that effective and sustainable mental health services were required, especially for bereaved single-child parents.
\end{abstract}

Keywords: earthquake, PTSD, depression, bereavement, prevalence 
Running title: PTSD and depression among bereaved earthquake survivors

\section{Introduction}

On the afternoon of May 12, 2008, an earthquake measuring 8.0 on the Richter scale hit Sichuan province in western China. Official state statistics reported a death toll of 69,227 with another 17,923 missing (State Council Information Office, 2008), of them 5,335 primary and secondary students were killed due the collapse of school buildings (Chen \& Hu, 2009). Around 4.8 million were left homeless. The dreadful feeling of loss coupled with the immense trauma of disaster could produce severe mental health consequences to all survivors; especially those who had suffered loss of their loved ones. The two most frequently reported psychiatric morbidities among disaster survivors in general (Basoglu, Kilic, Salcioglu, \& Livanou, 2004; Salcioglu, Basoglu, \& Livanou, 2007) and bereaved individuals in particular (Kaltman \& Bonanno, 2003) are PTSD and depression.

Numerous studies have examined the prevalence rates of PTSD and depression as well as their relevant risk factors among victims of different natural disasters. Some studies have suggested that the sudden unexpected death of a loved one is an important risk factor of PTSD (Basoglu et al., 2004; Basoglu, Salcioglu, \& Livanou, 2002; Breslau et al., 1998; Goenjian et al., 1994; Jia et al., 2010; Kun, Han, Chen, \& 
Yao, 2009; van Griensven et al., 2006) and depression (Livanou, Basoglu, Salcioglu, \& Kalendar, 2002; Salcioglu, Basoglu, \& Livanou, 2003; Salcioglu et al., 2007; van Griensven et al., 2006) among disaster survivors; however, studies on the magnitude of psychopathological symptoms among bereaved survivors are very limited. Only a handful of studies in the fields of disaster management and bereavement in general have reported the prevalence rates of PTSD and depression among bereaved survivors following sudden unexpected death of loved ones (Fullerton, Ursano, Kao, \& Bharitya, 1999; Goenjian et al., 2009; Kristensen, Weisaeth, \& Heir, 2009; Kuo et al., 2003; Schut, de Keijser, Van den Bout, \& Dijkhuis, 1991; Yuan et al., 2009). Even so, these studies were mainly conducted among general bereaved adults with different types of bereavement including loss of a parent, spouse, child, or sibling (Kristensen et al., 2009; Kuo et al., 2003; Morina, Rudari, Bleichhardt, \& Prigerson, 2010; Yuan et al., 2009), bereaved children who have lost their parents (Goenjian et al., 2009; Stoppelbein \& Greening, 2000), or bereaved adults who have lost their spouse due to accidents (Schut et al., 1991). The estimated rates of PTSD and depression among older bereaved people facing spousal loss have also been reported in the literature (Elklit \& O'Connor, 2005; O'Connor, 2010). Up to date, only one study (Murphy et al., 1999) has examined the estimated rate of probable PTSD among bereaved parents who have suffered sudden loss of their children. 
It is important to note that most of the aforementioned studies are conducted with population groups of developed countries. Only one study (Yuan et al., 2009) has been conducted in the unique socio-cultural environment of mainland China where single-child families form the majority due to the "one couple, one child" policy since the late 1970s. With downsized family networks as a result of population control, unexpected death of a loved one among Chinese families can be devastating and traumatic for survivors. While the differences in PTSD and depressive symptoms between bereaved and non-bereaved survivors after the Sichuan earthquake (also known as Wenchuan earthquake according to location of the epicenter) have been compared in the prior study (Yuan et al., 2009), the differences in psychopathological symptoms among different types of bereavement remain unclear. Hence, an examination of the estimated rates of psychopathological symptoms among different bereaved survivors would prove invaluable for the development and implementation of culturally-sensitive, effective, and sustainable mental health services for them.

Among the bulk of research that has examined the predictors of post-disaster psychiatric morbidities, the most frequently reported risk factors of PTSD following earthquakes include female gender, older age, low level of education, previous psychiatric illness, degree of exposure to earthquake, unexpected death of loved ones, loss of friends and neighbors, loss of property, loss of resources, financial loss, 
disruption in life, trapped under rubble, greater fear during the earthquake, number of traumatic experiences, and lack of governmental support (Basoglu et al., 2004; Breslau et al., 1998; Chang, Connor, Lai, Lee, \& Davidson, 2005; Salcioglu et al., 2007). In terms of post-disaster depression, the reported risk factors include geographic location, suffering from significant loss, female gender, being directly affected by the disaster, experiencing panic attack or multiple life stressors, having been exposed to previous traumatic events, ongoing socioeconomic adversity, death of loved ones, and previous psychiatric illnesses (Armenian et al., 2002; Kilic \& Ulusoy, 2003; Nandi, Tracy, Beard, Vlahov, \& Galea, 2009; Person, Tracy, \& Galea, 2006). While the unexpected death of a loved one has repeatedly been found as a significant risk factor for PTSD and depression, the impact of different types of bereavement on the severity of PTSD and depressive symptoms, especially among different ethnics groups, remains largely unclear. Although it has been suggested that the loss of a child generally elicit more intense and complicated grief reactions than other types of bereavement due to both the parental bereavement experience itself and the nature of parent-child bonding (Wijngaards-de Meij et al., 2007), studies on the associations between the type of bereavement and the severity of PTSD and depressive symptoms are rare.

In a recent study, our research team has examined the association between the 
type of bereavement and PTSD symptoms severity among survivors following the Sichuan earthquake in mainland China (Chan et al., 2011). We found that loss of a child was a strong predictive factor of PTSD symptoms for the parents. Although we have not examined the associations of different types of bereavement with depressive symptoms severity in that study, it is not unreasonable to assume that the predictors for depression may be similar to those for PTSD based on the high reported correlation between PTSD and depressive symptoms among our sample.

Thus, the main purpose of the current study was to examine the prevalence rates of PTSD and depressive symptoms among different bereaved survivors compared to a control group of non-bereaved survivors following the Sichuan earthquake. The second aim of this study was to examine predictive factors of depressive symptoms severity among earthquake survivors. We hypothesized that the loss of a child is also a significant predictive factor of depressive symptoms severity among survivors. Though based on the same dataset, the current study was distinctive from our previous study (Chan et al., 2011) for that that study only examined the estimated rate of PTSD symptoms in two different communities, whereas the current study was the first to examine psychopathological symptoms among bereaved adults experiencing different types of bereavement within the unique socio-cultural context of mainland China, which would extend our previous findings and contribute to the body of knowledge 
for understanding of the psychiatric sequelae of disasters in particular, and of bereavement in general.

\section{METHOD}

\section{Sampling}

Participants of this study comprised of survivors of the 2008 Sichuan earthquake. Participant recruitment was conducted in two heavily affected areas within close proximity of the epicenter. The first site was the town of Guankou (20 km from the epicenter) - the cultural and economic center of Dujianyan City. This town was selected for one of our psychological relief team was stationed there to provide psychological assistance to the earthquake survivors. The second site was the town of Jiannan (45 km from the epicenter) - the cultural and economic center of Mianzu City. This town was selected because it was the largest temporary camp community close to the epicenter. These two areas were seriously affected where the number of students died due to collapsing school buildings was only secondary to those in Beichuan county town, the most seriously damaged area during the earthquake. The two towns shared similar socioeconomic status before the earthquake.

Household was used in this study as the primary sampling unit, which was defined as all members within a family living together. To reduce sampling bias, only 
one competent member (those who can read and understand all questions of the study protocol or can communicate well with interviewers) within each household was selected by chance as the participant. The inclusion criteria included those who were competent and have experienced the earthquake. Those who did not experience the earthquake or suffered from dementia, mental retardation or severe mental disorders (e.g., schizophrenia) were excluded. To minimize the impact of other traumatic events unrelated to the earthquake, those with physical disabilities not resulting from the earthquake were also excluded. No restriction in age was set initially; but in light to reduce the likelihood of bias, those minors under the age of 18 years were not included in data analysis.

At the town of Guankou, cluster sampling was conducted in a temporary camp community, which was one of the earliest temporary camp communities in the town. Of the 425 households in the community, 302 available households were contacted and 254 survivors were successfully interviewed, but 11 ineligible questionnaires with minors were excluded. Subsequently, 243 valid questionnaires (response rate, 80.5\%) were included for data analysis. At the town of Jiannan, stratified random sampling based on prefabricated buildings (50 to 55 households in each building) was conducted from all of 11 temporary camp communities in the town. There are generally 5 units in each building and 10 to 11 households in each unit. Only one 
household in each unit was randomly selected. Of the 1,819 households contacted, 1,654 survivors were successfully interviewed but 113 incomplete questionnaires and 69 ineligible questionnaires with minors were excluded. Accordingly, a total of 1,482 valid questionnaires (response rate, 81.5\%) were included for data analysis. Finally, the questionnaires collected from the two sites were pooled. Of all 1,725 valid questionnaires, 251 bereaved survivors (who suffered the loss of one or more family members or/and relatives) and 1,474 non-bereaved survivors were identified.

\section{Procedure}

Data collection in the town of Guankou was conducted in the mid December 2008, seven months after the earthquake; while data collection in the town of Jiannan was conducted in the mid January 2009, eight months after the earthquake. Each period of data collection lasted for one week. The interviewers in this study included trained psychiatrists, psychiatric nurses, graduate and postgraduate students who majored in psychology or social sciences. Prior to the interviews, either verbal or written informed consent was obtained from each participant, while the aim and significance of the survey were explained in detail. For those participants who had difficulties in understanding the protocol questions because of their limited level of education, interviewers helped them by asking the questions one at a time and then filling in the 
answers for them.

\section{Measures}

The socio-demographic information collected for this study include age, gender, level of education, marital status, number of sons and/or daughters, and living areas (urban, rural, or suburban).

Trauma-related experiences were assessed with the following items: (a) the impact of earthquake on their house (none, mild, moderate, severe, and totally collapsed); (b) if they had any friends, colleagues, or neighbors who died in the earthquake (no, yes); and (c) if they had lost any family member or relative in the earthquake (no, yes). Those who reported death of family members or relatives were asked additional questions to indicate their relationship with the deceased. The responses were then recoded into two dichotomous variables: death in the family (no, yes), and loss of second-degree relatives (no, yes). Death in the family was further recoded into four dichotomous variables: loss of a child or grandchild, loss of a parent or grandparent, loss of a spouse, and loss of a sibling. Past experience of bereavement was also coded (no, yes) if participants had ever lost any family member or relative before the earthquake.

PTSD symptoms were assessed using the Chinese version (Wu \& Chan, 2003; 
Chen, Lai, Liao, \& Lin, 2005) of the Impact of Event Scale-Revised (IES-R, Weiss \& Marmar, 1997), a self-report instrument comprising 22 items to assess PTSD symptoms after a specific traumatic stressor. The IES-R is one of the most widely used measures of PTSD symptoms in traumatic-related research and clinical settings (Elhai, Gray, Kashdan, \& Franklin, 2005). It consists of three subscales corresponding to the three dimensions of the DSM-IV criteria for PTSD (American Psychiatric Association, 1994); namely, intrusive, avoidance, and hyperarousal symptoms. Each participant was asked to indicate the frequency of their distress for each of the 22 symptoms on a four-point scale, and a non-standard scoring scheme was used $(0=n o t$ at all, 1 = seldom, $3=$ sometime, $5=$ often). A total score was calculated as the mean response across all items. The Chinese version has shown acceptable psychometric properties (Wu \& Chan, 2003; Chen, Lai, Liao, \& Lin, 2005).

Depressive symptoms were assessed using the 10-items Center for Epidemiological Studies Depression Scale (CES-D, Andresen, Malmgren, Carter, Patrick; 1994), which is a widely used instrument for screening probable clinical depression and measuring severity of depressive symptoms. The translated Chinese CES-D had been validated in a community sample (Boey, 1999). The short CES-D scale consists of 10 items rating on a scale from 0 to 3 based on the frequency of occurrences during the past week (not at all, rarely, sometimes, often). The total score 
equals to the summarized score of all items in the scale (ranges from 0 to 30).

\section{Data Analysis}

In this study, descriptive analyses were performed for socio-demographic variables, trauma-related indicators, IES-R total score, and CES-D total score, using $\chi^{2}$ and $t$ test to evaluate differences between the two groups. The percentages of clinically significant PTSD symptoms and depressive symptoms in the two groups were compared using $\chi^{2}$ test. Bivariate correlation analyses among all variables were also examined with both samples pooled. Finally, stepwise hierarchical multivariate regression analyses were performed with socio-demographic and trauma-related variables serving as independent variables and the severity of depressive symptoms indicated by the CES-D total score serving as the dependent variable, whilst adjusting for clustering of sampling sites. In the first step, all independent variables including socio-demographic variables (age, gender, education, marital status, number of sons/daughters, and living area) and trauma-related indicators (house damage, loss of friends or neighbors, loss of a child, loss of a spouse, loss of a parent, loss of a sibling,

loss of $2^{\text {nd }}$ degree relatives, and past experience of bereavement) were entered into the regression model to examine the associations between independent variables and depressive symptoms severity. In the second step, the IES-R total score was entered 
into the regression model to examine if the effects of potential risk factors on the severity of depressive symptoms could be mediated by PTSD symptoms. To determine the unique contribution of each independent variable, the squared semipartial correlation coefficients $\left(\mathrm{sr}^{2}\right)$ were also examined. All analyses were conducted with SPSS (Version 18.0 for Windows). A value of $p<.05$ was considered statistically significant for regression analyses, and a Bonferroni corrected $p$ value ( $p$ $=0.05 / 20=0.0025)$ was employed for multiple tests.

\section{Results}

The sociodemographic characteristics and trauma-related indicators of the bereaved survivors group and the non-bereaved survivors group are presented in Table 1. As shown, there was no significant difference in age, sex, marital status, levels of education, living areas, and the extent of residential house damage between the two groups using the Bonferroni corrected $p$ value. The proportion of participants with one son/daughter is higher in the bereaved group compared to the non-bereaved group, but the difference is not significant with the Bonferroni corrected $p$ value. Compared to the non-bereaved group, the bereaved group had significantly higher proportion of participants coming from the town of Guankou $(\mathrm{p}<0.001)$ and significantly more past experiences of bereavement $(p<.001)$. 
In this study, a score of 2.0 or higher across all items of the IES-R was used to define an estimate rate of clinically significant PTSD symptoms. This cut-point was reported to have a sensitivity of .89 and a specificity of .90 (Wohlfarth, van den Brink, Winkel, \& ter Smitten, 2003). According to this criterion, 50.2\% of bereaved survivors and $27.1 \%$ non-bereaved survivors had clinically significant PTSD symptoms (Table 2); the difference between the two groups was significant $\left(\chi^{2}=\right.$ 53.82, $p<.001$ ). Specifically, $65.6 \%$ of survivors who lost first-degree family members had clinically significant PTSD symptoms; while those who lost a child represented the highest proportion (80\%) of participants with clinically significant PTSD symptoms.

A threshold of 12 for the total score of the 10-items CES-D recommended by Cheng and Chan (2005) was used to define an estimated rate of clinically significant depressive symptoms. According to this criterion, 55.8\% of bereaved survivors and 37.5\% non-bereaved survivors had clinically significant depressive symptoms (Table $2)$; the difference between the two groups was significant $\left(\chi^{2}=29.75, p<.001\right)$. Specifically, $64.8 \%$ of survivors who lost first-degree family members had clinically significant depressive symptoms, and those who lost a child represented the highest proportion (81.8\%) of participants with clinically significant depressive symptoms. 
As shown in Table 3, results of multivariate regression analyses indicated that the depressive symptoms severity was significantly associated with proximity to the epicenter, female gender, separated or divorced marital status, living in rural or suburban areas, the extent of residential house damage, loss of friends or neighbors, loss of a child, and loss of a parent after adjusting for socio-demographic variables. As indicated by the squared semi-partial correlation coefficients in Table 3, the amount of variance explained ranged from $0.3 \%$ for living areas to $3.0 \%$ for proximity to the epicenter. After the IES-R total score was entered into the regression model, the significant relationships were substantially reduced or became insignificant for trauma-related variables in addition to female gender, as indicated by the changes of beta values. Age, education, number of sons/daughters, loss of a spouse, loss of a sibling, loss of $2^{\text {nd }}$-degree relatives, and past experience of bereavement were not statistically significantly associated with depressive symptoms severity.

\section{Discussion}

This study is one of a few studies having examined the estimated rates of clinically significant PTSD and depressive symptoms in bereaved adults who have suffered sudden unexpected death of loved ones. Our results indicated that the estimated rates of clinically significant PTSD and depressive symptoms among 
bereaved survivors approximately 7.5 months after the 2008 Sichuan earthquake were $50.2 \%$ and $55.8 \%$ respectively, compared to $27.1 \%$ and $37.5 \%$ among non-bereaved earthquake survivors. These results were comparable to those reported in a prior study (Yuan et al., 2009). Our results further indicated that the estimated rates of PTSD and depressive symptoms among those who suffered the loss of first-degree family members (65.6\% and $64.8 \%$ respectively) were much higher than those who lost second-degree relatives ( $34.1 \%$ and $45.5 \%$ respectively). These results suggest that PTSD and depressive symptoms are prevalent in bereaved survivors, especially those who have suffered loss of first-degree family members, and that psychiatric sequelae of the intersection of trauma with bereavement is severe.

Due to the disparity of measurements and differences in time of assessment, sample size, and sampled population, comparing our results with those reported in previous studies of bereaved individuals following disasters or accidents should be cautious. For example, using face-to-face structured clinical interviews, Kristensen et al. (2009) reported that the prevalence rates of PTSD and depression among the directly exposed bereaved individuals two years after the 2004 tsunami disaster were 34.4\% and 25\% respectively. Kuo et al. (2003) investigated the prevalence of PTSD and major depression among 120 bereaved survivors aged 12 to 84 years old two months after a severe earthquake in Taiwan and reported that the prevalence rates of 
PTSD and depression were 37\% and 16\% respectively. Morina et al. (2010) examined the rates of PTSD and depression among 60 bereaved individuals who had lost first-degree relatives due to war-related violent in Kosovo in 1998/1999 and reported that 55.0\% fulfilled the criteria for PTSD and 38.3\% for depression. Furthermore, an earlier study (Fullerton et al., 1999) on bereaved individuals two months following a plane crash showed a significantly higher rate of depression (35\%) compared with a comparison group of non-bereaved (10\%). Such variations in findings across studies may well highlight the imperative need to consider the contextual and socio-cultural factors in the psychiatric outcomes of disasters and bereavement.

A critical finding in this study might be that higher rates of PTSD symptoms (80.0\%) and depressive symptoms (81.8\%) were observed among bereaved parents who had experienced the unexpected loss of their child. Although we could not compare the estimated rate of depressive symptoms in our study with other research since no similar results were found in literature, our estimated rate of PTSD symptoms among bereaved parents was much higher than the reports in a previous study (Murphy et al., 1999) that the prevalence rate of probable PTSD was $40 \%$ for bereaved mothers and $14 \%$ for bereaved fathers 4 months after the violent deaths of their 12-to-28-year-old children in a community-based sample of 261 bereaved parents (171 mothers and 90 fathers). Apart from variability in measurements, 
perhaps the most important reason attributing to such vast difference is the fact that the majority of bereaved parents in our study have lost their only child due to collapsing school buildings during the earthquake. In modern society, children have become the major source of joy, hope, meaning and purpose in the life for all parents, and the death of a child is utmost devastating (Hindmarch, 2009). With the unique socio-cultural environment in mainland China, sudden loss of a child is particularly traumatizing and heartbreaking for reasons beyond the 'one couple, one child' policy. In traditional Chinese societies, Confucian filial piety (hsiao) predominates (Bond, 1987, Holroyd \& Mackenzie, 1995), and family plays important roles in defining one's status in society, maintaining the livelihood of individual family members, as well as providing care and support to the elderly (Lee, 1998). Children are also a key element in ensuring family linkage and generational continuity. Hence, parents who have suffered sudden loss of their only child may feel extremely desperate especially for those who could no longer conceive. These bereaved parents are highly vulnerable to PTSD and depressive symptoms. Therefore, appropriate and culturally-sensitive psychological assistance and mental health services should be directed to this particular group of bereaved survivors.

Our previous study indicated that the loss of a child was a strong predictor of PTSD symptoms severity among survivors (Chan et al., 2011). Results of multivariate 
regression analysis in the current study further revealed that the loss of a child was a strong predictor of depressive symptoms severity among adult survivors. Compared to other types of family bereavement and other types of loss including loss of friends or neighbors and residential house damage or collapse, the squared semi-partial correlation coefficient was highest for the loss of a child $\left(\mathrm{sr}^{2}=.023\right)$, suggesting that it is one of the most stressful and painful life event for survivors. Thus, our hypothesis is supported. To the best of our knowledge, no other study has examined the independent effects of different types of family bereavement, so we cannot compare our results with others in this regard. Nevertheless, our results confirm that the sudden loss of a child is the most traumatized and painful experience for parents compared with other types of bereavement, which can result in a broad range of psychiatric morbidities and somatic symptoms. Our results further indicated that the effects of loss of a child and residential house damage on depressive symptoms severity could not be fully mediated by PTSD symptoms, suggesting that loss of a child and residential house damage or collapse might have led to depression through other mechanisms such as prolonged grief and disruption of daily life following the disaster.

In the current study, insignificant association between the loss of a spouse and depressive symptoms may be due to insufficient number of survivors in this subgroup. Insignificant difference in depressive symptoms between the loss of a sibling and the 
loss of a second-degree relative was in line with previous reports (Fullerton et al., 1999). A significant association between the loss of friends or neighbors and depressive symptoms severity may be due to downsized social networks and less received social support after the earthquake. A previous study on disaster-related bereavement has reported that low perceived support from friends is a significant predictor of depression two months post-disaster (Fullerton et al., 1999). Consistent with previous findings (e.g., Chou et al., 2005), divorced/widowed status was significantly associated with the severity of depressive symptoms in the current study.

There are several limitations to this study. First, the rates of PTSD and depressive symptoms reported in our study are only estimations of the true prevalence rates in the population, since PTSD and depressive symptoms were measured with self-rated instruments that were not designed to estimate diagnostic prevalence of PTSD and depression. Thus, interpretation and generalization of the results in the current study should be cautious. Second, several factors were not included in this study, such as the experience of being trapped under rubble, severity of injury during the earthquake, property loss, post-disaster stressors, pre-disaster psychiatric morbidities, and perceived social support, but these factors have been well documented in other studies. Third, the sample in this study was recruited from two different county towns, which might have introduced sampling bias. 
In summary, the results of the current study showed that mental health problems were prevalent among Chinese bereaved adults seven to eight months after the 2008 Sichuan earthquake. The loss of a child was a significant predictor of depressive symptoms severity. Other predictors of the depressive symptoms severity included female gender, divorced or widowed status, living in rural areas, collapsed or severely damaged residential house, loss of friend or neighbor, loss of a parent, and close proximity to the epicenter. Effective and sustainable psychosocial assistance and mental health services are required and special attention should be directed to bereaved single-child parents following such a devastating natural disaster.

(4,306 words) 


\section{Acknowledgement}

Funding for this study was provided by the Sichuan Earthquake Roundtable

Fund Internal Seed Funding of the University of Hong Kong. The funding sources had no further role in study design; in collection, analysis and interpretation of data; in the writing of the report; and in the decision to submit the paper for publication. The authors wish to thank all those who helped to collect the data and those who kindly volunteered to participate in the study. The authors further wish to express their deepest condolences to all families and individuals who have suffered loss of family members and relatives during the 2008 Sichuan earthquake disaster. 


\section{References:}

American Psychiatric Association. (1994). Diagnostic and statistical manual of mental disorders (4th ed.). Washington, DC: Author.

Andresen, E. M., Malmgren, J. A., Carter, W. B., \& Patrick, D. L. (1994). Screening for depression in well older adults: evaluation of a short form of the CES-D (Center for Epidemiologic Studies Depression Scale). American Journal of Preventive Medicine, 10(2), 77-84.

Armenian, H. K., Morikawa, M., Melkonian, A. K., Hovanesian, A., Akiskal, K., \& Akiskal, H. S. (2002). Risk factors for depression in the survivors of the 1988 earthquake in Armenia. Journal of Urban Health, 79(3), 373-382.

Basoglu, M., Kilic, C., Salcioglu, E., \& Livanou, M. (2004). Prevalence of posttraumatic stress disorder and comorbid depression in earthquake survivors in Turkey: an epidemiological study. Journal of Traumatic Stress, 17(2), $133-141$.

Basoglu, M., Salcioglu, E., \& Livanou, M. (2002). Traumatic stress responses in earthquake survivors in Turkey. Journal of Traumatic Stress, 15(4), 269-276.

Boey, K. W. (1999). Cross-validation of a short form of the CES-D in Chinese elderly. International Journal of Geriatric Psychiatry, 14(8), 608-617.

Bond, M. (1987). Chinese values and the search for culture. Journal of Cross-Culture Psychology, 18, 143-164.

Breslau, N., Kessler, R. C., Chilcoat, H. D., Schultz, L. R., Davis, G. C., \& Andreski, P. (1998). Trauma and posttraumatic stress disorder in the community: the 1996 Detroit Area Survey of Trauma. Archives of General Psychiatry, 55(7), 626-632.

Chan, C. L. W., Wang, C. W., Qu, Z., Lu, B. Q., Ran, M. S., Ho, A. H. Y., et al. (2011) 
Posttraumatic Stress Disorder Symptoms Among Adult Survivors of the 2008

Sichuan Earthquake in China. Journal of Traumatic Stress, 24(3), 295-302.

Chang, C. M., Connor, K. M., Lai, T. J., Lee, L. C., \& Davidson, J. R. (2005).

Predictors of posttraumatic outcomes following the 1999 Taiwan earthquake. Journal of Nervous and Mental Disease, 193(1), 40-46.

Chen J., \& Hu Y. (2009, May 8). China issues student death toll from last year's quake. China Daily. Retrieved from: http://www.chinadaily.com.cn/china/2009wenchuan/2009-05/08/content_7755 348.htm

Chen, S. C., Lai, Y. H., Liao, C. T., \& Lin, C. C. (2005). Psychometric testing of the Impact of Event Scale-Chinese Version (IES-C) in oral cancer patients in Taiwan. Supportive Care in Cancer, 13(7), 485-492.

Cheng, S. T., \& Chan, A. C. (2005). The Center for Epidemiologic Studies Depression Scale in older Chinese: thresholds for long and short forms. International Journal of Geriatric Psychiatry, 20(5), 465-470.

Chou, F. H., Su, T. T., Chou, P., Ou-Yang, W. C., Lu, M. K., \& Chien, I. C. (2005). Survey of psychiatric disorders in a Taiwanese village population six months after a major earthquake. Journal of the Formosan Medical Association, 104(5), 308-317.

Elhai, J. D., Gray, M. J., Kashdan, T. B., \& Franklin, C. L. (2005). Which instruments are most commonly used to assess traumatic event exposure and posttraumatic effects?: A survey of traumatic stress professionals. Journal of Traumatic Stress, 18(5), 541-545.

Elklit, A., \& O'Connor, M. (2005). Post-traumatic stress disorder in a Danish population of elderly bereaved. Scandinavian Journal of Psychology, 46(5), 439-445. 
Fullerton, C. S., Ursano, R. J., Kao, T. C., \& Bharitya, V. R. (1999). Disaster-related bereavement: acute symptoms and subsequent depression. Aviation Space and Environmental Medicine, 70(9), 902-909.

Gleser, G. C., Green, B. L., \& Winget, C. N. (1981). Prolonged psychosocial effects of disaster: a study of Buffalo Creek. New York: Academic Press.

Goenjian, A. K., Najarian, L. M., Pynoos, R. S., Steinberg, A. M., Manoukian, G., Tavosian, A., et al. (1994). Posttraumatic stress disorder in elderly and younger adults after the 1988 earthquake in Armenia. American Journal of Psychiatry, 151(6), 895-901.

Goenjian, A. K., Walling, D., Steinberg, A. M., Roussos, A., Goenjian, H. A., \& Pynoos, R. S. (2009). Depression and PTSD symptoms among bereaved adolescents 6(1/2) years after the 1988 Spitak earthquake. Journal of Affective Disorders, 112(1-3), 81-84.

Hindmarch, C. (2009). On the death of a child (3rd ed). Abingdon, Oxon [England]: Radcliffe Publishing.

Holroyd, E. A., \& Mackenzie, A. E. (1995). A review of the historical and social processes contributing to care and caregiving in Chinese families. Journal of Advanced Nursing, 22(3), 473-479.

Jia, Z., Tian, W., Liu, W., Cao, Y., Yan, J., \& Shun, Z. (2010). Are the elderly more vulnerable to psychological impact of natural disaster? A population-based survey of adult survivors of the 2008 Sichuan earthquake. BMC Public Health, $10,172$.

Kaltman, S., \& Bonanno, G. A. (2003). Trauma and bereavement: Examining the impact of sudden and violent deaths. Journal of Anxiety Disorders, 17(2), $131-147$.

Kilic, C., \& Ulusoy, M. (2003). Psychological effects of the November 1999 
earthquake in Turkey: an epidemiological study. Acta Psychiatrica Scandinavica, 108(3), 232-238.

Kristensen, P. Æ., Weisae@Eth, L., \& Heir, T. (2009). Psychiatric disorders among disaster bereaved: An interview study of individuals directly or not directly exposed to the 2004 tsunami. Depression and Anxiety, 26(12), 1127-1133.

Kun, P., Han, S., Chen, X., \& Yao, L. (2009). Prevalence and risk factors for posttraumatic stress disorder: a cross-sectional study among survivors of the Wenchuan 2008 earthquake in China. Depression and Anxiety.

Kuo, C. J., Tang, H. S., Tsay, C. J., Lin, S. K., Hu, W. H., \& Chen, C. C. (2003). Prevalence of psychiatric disorders among bereaved survivors of a disastrous earthquake in taiwan. Psychiatric Services, 54(2), 249-251.

Lee, A. E. Y. (1998). Family social support patterns of the Chinese elderly in Beijing, Shanghai, Guangzhou and Los Angeles: The role of filial piety. Dissertation Abstracts International, 58, 3723.

Livanou, M., Basoglu, M., Salcioglu, E., \& Kalendar, D. (2002). Traumatic stress responses in treatment-seeking earthquake survivors in Turkey. Journal of Nervous \& Mental Disease, 190(12), 816-823.

Morina, N., Rudari, V., Bleichhardt, G., \& Prigerson, H. G. (2010). Prolonged grief disorder, depression, and posttraumatic stress disorder among bereaved Kosovar civilian war survivors: A preliminary investigation. International Journal of Social Psychiatry, 56(3), 288-297.

Murphy, S. A., Braun, T., Tillery, L., Cain, K. C., Johnson, L. C., \& Beaton, R. D. (1999). PTSD among bereaved parents following the violent deaths of their 12- to 28-year-old children: A longitudinal prospective analysis. Journal of Traumatic Stress, 12(2), 273-291.

Nandi, A., Tracy, M., Beard, J. R., Vlahov, D., \& Galea, S. (2009). Patterns and 
predictors of trajectories of depression after an urban disaster. Annals of Epidemiology, 19(11), 761-770.

O'Connor, M. (2010). PTSD in older bereaved people. Aging and Mental Health, 14(6), 670-678.

Person, C., Tracy, M., \& Galea, S. (2006). Risk factors for depression after a disaster. Journal of Nervous and Mental Disease, 194(9), 659-666.

Salcioglu, E., Basoglu, M., \& Livanou, M. (2003). Long-term psychological outcome for non-treatment-seeking earthquake survivors in Turkey. Journal of Nervous and Mental Disease, 191(3), 154-160.

Salcioglu, E., Basoglu, M., \& Livanou, M. (2007). Post-traumatic stress disorder and comorbid depression among survivors of the 1999 earthquake in Turkey. Disasters, 31(2), 115-129.

Schut, H. A., de Keijser, J., Van den Bout, J., \& Dijkhuis, J. H. (1991). Post-traumatic stress symptoms in the first years of conjugal bereavement. Anxiety Research, 4(3), 225-234.

State Council InformationOffice of the People’s Republic ofChina. (2008, September 26). Latest developments of Wenchuan earthquake relief by September 25, 2008. Retrieved from http://www.scio.gov.cn/zxbd/wz/200905/t310218.htm Stoppelbein, L., \& Greening, L. (2000). Posttraumatic stress symptoms in parentally bereaved children and adolescents. Journal of the American Academy of Child \& Adolescent Psychiatry, 39(9), 1112-1119.

van Griensven, F., Chakkraband, M. L., Thienkrua, W., Pengjuntr, W., Lopes Cardozo, B., Tantipiwatanaskul, P., et al. (2006). Mental health problems among adults in tsunami-affected areas in southern Thailand. JAMA, 296(5), 537-548.

Weiss, D. S., \& Marmar, C. R. (1997). The impact of Event Scale - Revised In J. P. Wilson \& T. M. Keane (Eds.), Assessing psychological trauma and PTSD: A 
practitioner's handbook (pp. 399-411). New York: Guilford Press.

Wijngaards-de Meij, L., Stroebe, M., Schut, H., Stroebe, W., van den Bout, J., van der Heijden, P. G. M., et al. (2007). Patterns of Attachment and Parents' Adjustment to the Death of Their Child. Personality and Social Psychology Bulletin, 33(4), 537-548.

Wohlfarth, T. D., van den Brink, W., Winkel, F. W., \& ter Smitten, M. (2003). Screening for Posttraumatic Stress Disorder: an evaluation of two self-report scales among crime victims. Psychological Assessment, 15(1), 101-109.

Wu, K. K., \& Chan, K. S. (2003). The development of the Chinese version of Impact of Event Scale--Revised (CIES-R). Social Psychiatry and Psychiatric Epidemiology, 38(2), 94-98.

Yuan, Y., Mao, W. -J., Yang, D. -H. Ran, M. -S., Kong, D., Zhang, T., et al. (2009). [Comparison of the PTSD symptoms, depression and anxiety between bereaved and non-bereaved survivors after Wenchuan earthquake]. Chinese Journal of Behavioral Medicine and Brain Science, 18(12), 1109-1111. 
Table 1 Comparison of demographic characteristics between bereaved and non-bereaved

\begin{tabular}{|c|c|c|c|c|c|c|c|c|c|c|}
\hline & \multicolumn{4}{|c|}{ Bereaved $(n=251)$} & \multicolumn{4}{|c|}{ Non-Bereaved $(\mathrm{n}=$} & \multirow{2}{*}{$\operatorname{tor} x^{2}$} & \multirow{2}{*}{$p$} \\
\hline & $M$ & $S D$ & $n$ & $\%$ & $M$ & $S D$ & $n$ & $\%$ & & \\
\hline Age & 47.81 & 15.43 & & & 50.53 & 16.04 & & & 2.45 & .014 \\
\hline Gender & & & & & & & & & 2.29 & .130 \\
\hline Male & & & 98 & 39.0 & & & 651 & 44.2 & & \\
\hline Female & & & 153 & 61.0 & & & 823 & 55.8 & & \\
\hline Marital status & & & & & & & & & 2.18 & .536 \\
\hline Unmarried & & & 15 & 6.0 & & & 118 & 8.0 & & \\
\hline Married/remarried & & & 204 & 81.3 & & & 1142 & 77.5 & & \\
\hline Divorced or separated & & & 11 & 4.4 & & & 82 & 5.6 & & \\
\hline Widowed & & & 21 & 8.4 & & & 132 & 9.0 & & \\
\hline Education & & & & & & & & & 5.01 & .286 \\
\hline No formal education & & & 21 & 8.4 & & & 149 & 10.1 & & \\
\hline Elementary or below & & & 48 & 19.1 & & & 357 & 24.2 & & \\
\hline Middle or high school & & & 141 & 56.2 & & & 737 & 50.0 & & \\
\hline College or above & & & 38 & 15.1 & & & 219 & 14.9 & & \\
\hline No. of sons/daughters & & & & & & & & & 9.14 & $<.010$ \\
\hline 0 & & & 16 & 6.4 & & & 156 & 10.6 & & \\
\hline 1 & & & 163 & 64.9 & & & 816 & 55.4 & & \\
\hline 2 & & & 68 & 27.1 & & & 498 & 33.8 & & \\
\hline 3 or more & & & 4 & 1.6 & & & 4 & .3 & & \\
\hline Sites & & & & & & & & & 40.05 & $<.001$ \\
\hline Guankou Town & & & 68 & 27.1 & & & 175 & 11.9 & & \\
\hline Jiannan Town & & & 183 & 72.9 & & & 1299 & 88.1 & & \\
\hline
\end{tabular}


Living areas

$.20 \quad .655$

Urban area

$197 \quad 80.7$

115979.5

Rural or suburban area

$47 \quad 19.3$

$299 \quad 20.5$

Residential house damage

$6.28 \quad .179$

None

4

1.7

$15 \quad 1.1$

Mild

$2 \quad 0.8$

$47 \quad 3.4$

Moderate

$45 \quad 18.6$

$215 \quad 15.6$

Severe

$130 \quad 53.7$

$746 \quad 54.1$

Totally collapsed

$61 \quad 25.2$

$355 \quad 25.8$

Loss of friends/neighbors

$95 \quad 37.8$

$\begin{array}{llll}494 & 33.5 & 1.79 & .181\end{array}$

Loss of family members

$125 \quad 49.8$

Loss of a child

$55 \quad 21.9$

Loss of a spouse

$14 \quad 5.6$

Loss of a parent

$28 \quad 11.2$

Loss of a sibling

$33 \quad 13.1$

Loss of $2^{\text {nd }}$-degree relatives*

$140 \quad 55.8$

Past bereavement

$61 \quad 24.3$

$20213.7 \quad 18.60<.001$

IES - R total

$2.08 \quad 1.14$

$1.53 \quad 0.89$

$8.71<.001$

CES-D total

12.927 .11

$9.91 \quad 6.04$

$7.08<.001$

* The number includes those who have lost both a $1^{\text {st }}$-degree family member and one or more second-degree relatives.

IES-R: Impact Event Scale - Revised; CES-D: Center for Epidemiological Studies Depression Scale. 
Table 2. Comparison of PTSD symptoms and depressive symptoms between bereaved and non-bereaved

\begin{tabular}{ccccc}
\hline & \multicolumn{2}{c}{ PTSD symptoms } & \multicolumn{2}{c}{ Depressive symptoms } \\
& $n$ & $\%$ & $n$ & $\%$ \\
\hline Bereaved group $(\mathrm{n}=251)$ & 126 & 50.2 & 140 & 55.8 \\
Loss of family members $(\mathrm{n}=125)^{\mathrm{a}}$ & 82 & 65.6 & 81 & 64.8 \\
$\quad$ Loss of a child $(\mathrm{n}=55)$ & 44 & 80.0 & 45 & 81.8 \\
$\quad$ Loss of a spouse $(\mathrm{n}=13)^{\mathrm{b}}$ & 8 & 61.5 & 9 & 69.2 \\
$\quad$ Loss of a parent $(\mathrm{n}=25)^{\mathrm{b}}$ & 17 & 68.0 & 12 & 48.0 \\
$\quad$ Loss of a sibling $(\mathrm{n}=32)^{\mathrm{b}}$ & 13 & 40.6 & 15 & 46.9 \\
Loss of 2nd-degree relatives $(\mathrm{n}=123)^{\mathrm{c}}$ & 42 & 34.1 & 56 & 45.5 \\
Non-bereaved group $(\mathrm{n}=1474)^{2}$ & 400 & 27.1 & 553 & 37.5 \\
\hline
\end{tabular}

PTSD: Posttraumatic stress disorder;

a. The number includes those who have lost both first-degree family members and second-degree relatives.

b. The number does not include those who have also lost a family member in previous categories.

c. The number does not include those who have lost both second-degree relatives and first-degree family members. 
Table 3. Stepwise multiple regression analyses of the contribution of independent variables to depressive symptoms severity $(N=1,725)$

\begin{tabular}{lcccc}
\hline & \multicolumn{2}{c}{ Step 1: $\mathrm{R}^{2}=0.113^{\mathrm{f}}$} & \multicolumn{2}{c}{ Step 2: $\mathrm{R}^{2}=0.463^{g}$} \\
& Beta & $s r^{2}$ & \multicolumn{1}{c}{ Beta } & $s r^{2}$ \\
\hline Sites $^{\mathrm{a}}$ & $-.180^{* * *}$ & .030 & $-.085^{* * *}$ & .006 \\
Gender $^{\mathrm{b}}$ & $.109^{* * *}$ & .012 & .031 & - \\
Marital status $^{\mathrm{c}}$ & $-.123^{* * *}$ & .015 & $-.102^{* * *}$ & .011 \\
Living areas $^{\mathrm{d}}$ & $-.055^{*}$ & .003 & $-.046^{*}$ & .002 \\
House damage & $.091^{* * *}$ & .008 & $.045^{*}$ & .002 \\
Loss of friends or neighbors $^{\mathrm{e}}$ & $.051^{*}$ & .003 & .035 & - \\
Loss of a child & & .023 & $.053^{* *}$ & .003 \\
Loss of a parent & $.155^{* * *}$ & .023 & .002 & - \\
IES-R total & $.048^{*}$ & .002 & .628 & .364 \\
\hline
\end{tabular}

Note: Dependent variable $=$ Center for Epidemiological Studies Depression Scale (CES-D)

total score; IES-R: Impact Event Scale - Revised.

${ }^{\mathrm{a}} 1$ = Guankou Town, 2 = Jiannan Town;

${ }^{\mathrm{b}} 1$ = male, 2 = female;

${ }^{\mathrm{c}} 0=$ unmarried/separated/widowed, 1 = married;

${ }^{\mathrm{d}} 0=$ not urban, 1 = urban;

${ }^{\mathrm{e}} 0$ = none, 1 = yes;

${ }^{\mathrm{f}} F=25.08, p<.001 .{ }^{\mathrm{g}} F=150.10, p<.001$.

${ }^{*} p<.05 ;{ }^{* *} p<.01 ;{ }^{* * *} p<.001$ 